\title{
O uso da quimioterapia no tratamento do retinoblastoma: Avaliação retrospectiva
}

\author{
The use of chemotherapy in the treatment of retinoblastoma:Retrospective analysis
}

Fernando Leite Kronbauer ${ }^{(1)}$

Zélia Maria S. Corrêa ${ }^{(2)}$

Cláudia Tyllmannn ${ }^{(1)}$

Carlos Eug'ênio Escovar ${ }^{(3)}$

Ítalo Mundialino Marcon ${ }^{(4)}$
Estudo realizado no Serviço de Oftalmologia da Santa Casa de Misericórdia de Porto Alegre e na Unidade Oncológica do Hospital da Criança Santo Antônio.

Este trabalho foi parcialmente apresentado como Tema Livre no II Congresso da Sociedade Brasileira de Retina e Vítreo, Maio/99, Porto Alegre (RS).

Médico Residente do Serviço de Oftalmologia da Santa Casa de Misericórdia de Porto Alegre (RS).

(2) Chefe do setor de Oncologia Oftalmológica do Serviço de Oftalmologia da Santa Casa de Misericórdia de Porto Alegre (RS)

3) Departamento de Oncologia Pediátrica do Hospital da Criança Santo Antônio. Porto Alegre (RS).

(4) Chefe do Serviço de Oftalmologia da Santa Casa de Misericórdia de Porto Alegre, Professor Regente da Disciplina de Oftalmologia da Fundação Faculdade Federal de Ciências Médicas de Porto Alegre (RS). Os autores não possuem interesse financeiro de qualquer ordem no presente estudo.

Endereço para correspondência: Zélia M. Corrêa Av. Nilo Peçanha, 2421 - Porto Alegre (RS) CEP 91330-001. Fone/Fax: (51)328-5164. E-mail zmcorrea@zaz.com.br

\begin{tabular}{|c|}
\hline RESUMO \\
\hline 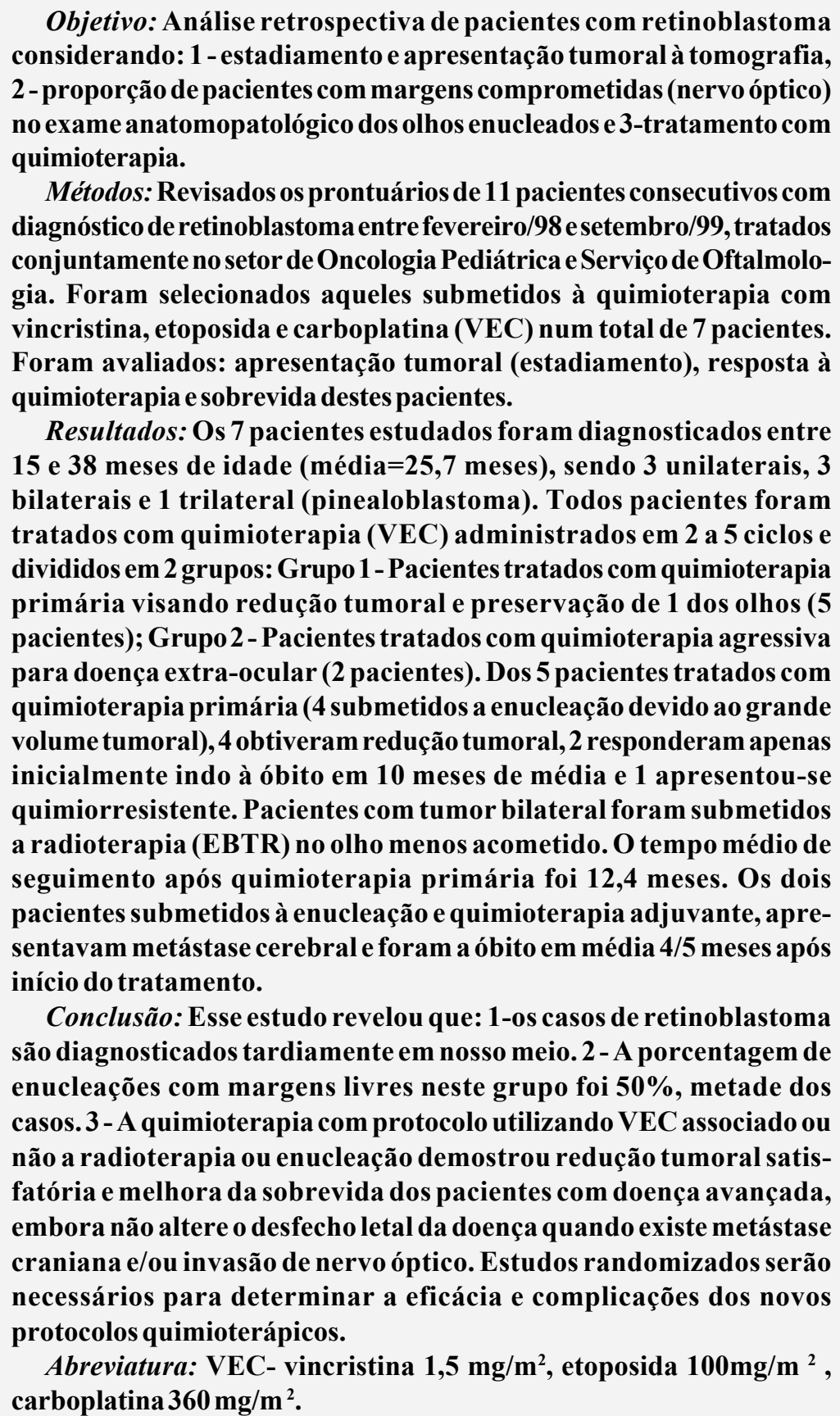 \\
\hline
\end{tabular}

Palavras-chave: Retinoblastoma: quimioterapia; Terapêutica. 


\section{INTRODUÇÃO}

Retinoblastoma é o tumor ocular mais freqüente na infância, e o segundo mais comum em todas as faixas etárias, com prevalência de 1:18.000 nascidos vivos nos EUA. No Brasil, a incidência relatada é de 5,9 casos por milhão de habitantes em São Paulo ${ }^{1,2}$. Não há preferência por sexo ou raça, podendo ocorrer de forma esporádica ou hereditária, sendo encontrado os dois olhos afetados em aproximadamente 1 a cada 3 casos $^{3}$.

A idade média ao diagnóstico é 18 meses. Apesar do retinoblastoma poder apresentar-se ao nascimento, a grande maioria dos casos é diagnosticado antes dos 3 anos de idade e raramente após os 6 anos de idade. Crianças com envolvimento bilateral apresentam sinais e sintomas mais precocemente do que aquelas com tumor unilateral, e costumam desenvolver tumores não oculares: pinealoblastoma ${ }^{4}$ (tumor trilateral) e sarcoma osteogênico ${ }^{5}$. O diagnóstico precoce e o pronto tratamento contribuem significativamente para a sobrevivência do paciente e conservação do globo ocular ${ }^{6}$.

Novas opções terapêuticas para o retinoblastoma ter surgido nos últimos anos, cada uma delas com riscos, benefícios e limitações particulares a serem consideradas. A enucleação do olho envolvido tem sido o método de escolha para os casos unilaterais devido à ausência de efeitos colaterais durante o tratamento e a longo prazo. Quando há comprometimento bilateral mas restrito ao globo ocular, ou unilateral com invasão do nervo óptico sugere-se a associação de radioterpia e quimioterapia para preservar o olho com possibilidade de visão ${ }^{8}$. Fotocoagulação e Crioterapia podem ser utilizados para tumores pequenos e circunscritos $(<3 \mathrm{~mm}$ de diâmetro) e sem invasão vítrea ${ }^{4}$.

A quimioterapia está indicada em 3 situações: 1- tumores grandes que não podem ser irradiados ou fotocoagulados, 2comprometimento vítreo e/ou do nervo óptico, e 3- doença extra-ocular.

A quimioterapia isolada não é suficiente para completo controle da doença. Após 2 meses de quimioterapia com 2 ciclos, existe um decréscimo de $35 \%$ na base do tumor e $50 \%$ na sua espessura, sendo necessário tratamento complementar para completa erradicação do tumor. O número de ciclos quimioterápicos a ser usado varia entre 2 a 6 ciclos de acordo com as particularidades de cada caso. Enquanto não existem estudos científicos padronizando o número de ciclos de quimioterapia, devem ser lembrados os efeitos a longo prazo que tal tratamento pode acarretar, e sua necessidade de terapia adjuvante ${ }^{9}$.

O protocolo quimioterápico desenvolvido no Children's Hospital da Universidade da Pensilvânia (Filadélfia, EUA), publicado por Shields e colaboradores ${ }^{9}$, utilizando vincristina, etoposide e carboplatina tem obtido resultados animadores mesmo em casos de doença avançada.

O objetivo deste trabalho foi avaliar, em pacientes com retinoblastoma, tratados com VEC : 1-estadiamento e apresen- tação tumoral à tomografia, 2-proporção de pacientes com envolvimento do nervo óptico em exame anatomo-patológico dos olhos enucleados e 3 -tratamento com quimioterapia como nova opção terapêutica..

\section{PACIENTES E MÉTODOS}

Foram revisados os prontuários de 11 pacientes consecutivos com diagnóstico de retinoblastoma tratados conjuntamente no setor de Oncologia Pediátrica e Serviço de Oftalmologia, no período de Fevereiro/98 a Setembro/99. Destes, 07 pacientes foram submetidos à quimioterapia com VEC como parte de seu tratamento.

Foram avaliados no estudo:

1- A proporção de sexos, uni, bi ou trilateralidade do tumor, e história familiar.

2- Apresentação na primeira consulta.

3- Média de idade de chegada ao Serviço dos casos unilaterais e bilaterais.

4- Apresentação na tomografia computadorizada e estadiamento segundo os critérios de Reese-Ellsworth ${ }^{1,12,17}$.

5- Tratamentos distribuídos de acordo com tumor restrito ao globo ocular (grupo1) ou doença extra-ocular no momento do diagnóstico (grupo2): Grupo I quimioterapia primária (número de ciclos) e enucleação, associando radioterapia (EBRT) 5.000 Gy diários 30 dias nos olhos menos comprometidos de pacientes com tumor bilateral com o objetivo de preservar um dos orgãos. Grupo II enucleação e quimioterapia adjuvante (número de ciclos). Na quimioterapia foi utilizado o protocolo com Vincristina $1,5 \mathrm{mg} / \mathrm{m}^{2}$, Etoposide $100 \mathrm{mg} / \mathrm{m}^{2}$ e Carboplatina $360 \mathrm{mg} / \mathrm{m}^{2}$.

6- Proporção de olhos com envolvimento do nervo óptico e presença de margens livres em exame anatomopatológico dos pacientes enucleados.

7- Sobrevida destes pacientes, e proporção de pacientes mortos pela doença associando ao estadiamento do tumor.

\section{RESULTADOS}

1- Sete pacientes portadores de retinoblastoma foram tratados com quimioterapia durante o período de Fevereiro/98 a Setembro/99, 4 eram do sexo masculino. Três casos eram unilaterais 3 bilaterais e 1 trilateral (Fig.1.A e 1.B, caso $\mathrm{n}^{\circ} 7$ ). Nenhum dos pacientes tinha história familiar de retinoblastoma.

2 - As queixas que levaram estes pacientes ao consultório médico foram: 2 por leucocoria, 2 por estrabismo, 2 por proptose e 1 por baixa acuidade visual. Em todos os casos, sinais e sintomas foram notados pelos familiares.

3 - A idade média de início do tratamento de todos os grupos foi de 25,7 meses (sendo o mais precoce com 15 meses e o mais tardio com 38 meses), os casos unilaterais e bilaterais foram diagnosticados em média aos 23,6 meses de idade, o 


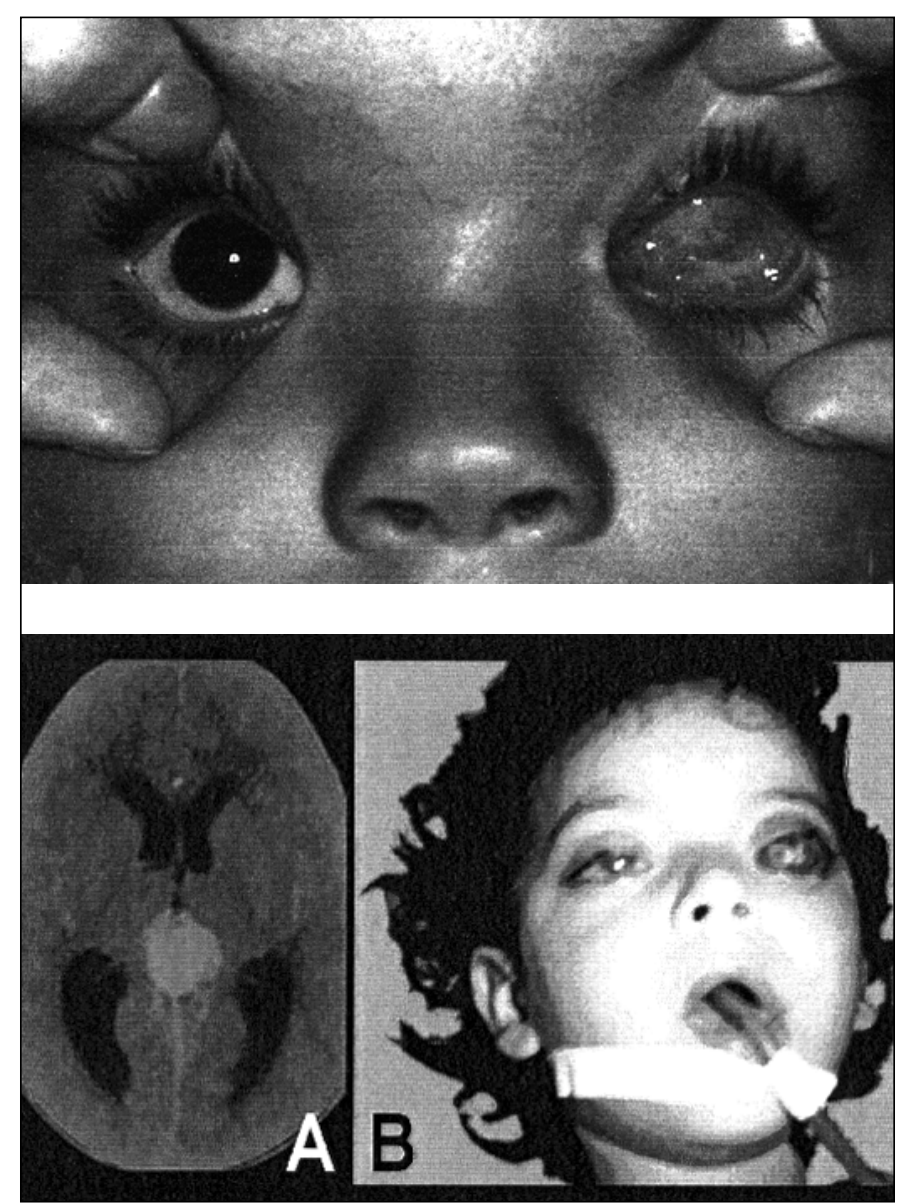

Figura 1 - Paciente $\mathrm{N}^{\circ} 7$ com retinoblastoma diagnosticado como trilateral. A. Tomografia computadorizada revela lesão tumoral com formato arredondado (pinealoblastoma). B. Ao exame externo percebe-se proptose bilateral, com exposição ocular, sendo o olho esquerdo mais proeminente com conteúdo tumoral necrótico visível, devido ao grande volume tumoral. único caso trilateral (com pinealoblastoma associado) foi o mais tardio, diagnosticado aos 38 meses de idade.

4 - A tomografia computadorizada revelou que 3 pacientes tinham tumor restrito ao globo e órbita e 4 com metástase em crânio. Dois pacientes desenvolveram metástase após início do tratamento com quimioterapia primária. $\mathrm{O}$ estadiamento segundo a classificação de Reese-Ellsworth foi feita para cada um dos olhos $(\mathrm{n}=11)$. Destes 11 olhos acometidos, 5 apresentavam estadiamento $\mathrm{Vb}, 4$ apresentavam $\mathrm{Va}$, e somente $1 \mathrm{com}$ IVa e 1 com IIIa. Todos os pacientes apresentavam pelo menos 1 olho classificado no grupo V (Tabela).

5 - Os pacientes foram distribuídos de acordo com a presença ou não de doença extra-ocular no momento do diagnóstico: Grupo I- Cinco pacientes receberam quimioterapia como tratamento primário, 4 obtiveram redução tumoral sendo que 2 responderam apenas inicialmente indo a óbito em 10 meses de média. $\mathrm{O}$ número de ciclos de quimioterapia usado foi em média de 3 (variando de 2 a 5 ciclos). O tempo médio de seguimento após quimioterapia primária foi de 12,4 meses (variação 6-18 meses) até o presente momento. Dois pacientes desenvolveram metástase após início da quimioterapia primária. Quatro pacientes foram submetidos à enucleação do pior olho, ou olho preenchido por tumor. Um paciente (Fig.2, caso $n^{\circ} 5$ ) apresentou sementes vítreas durante o tratamento com quimio e laser (termo-terapia), como já havia sido enucleado o olho pior (grupoV), necessitou tratamento complementar com radioterapia (EBRT). Atualmente, 4 meses pós EBRT, apresenta regressão tipo 2 das lesões intraoculares e visão 20/25. Outro paciente (caso $n^{\circ} 4$ ) tratado com radioterapia (EBRT) no olho menos acometido, foi submetido a enucleação do segundo olho devido a descolamento da

\begin{tabular}{|c|c|c|c|c|c|c|c|c|}
\hline \multicolumn{9}{|c|}{ Tabela. Pacientes com retinoblastoma tratados com Quimioterapia (VEC) e suas características individuais } \\
\hline $\begin{array}{l}\text { Idade do tratam. } \\
\text { inicial (meses) }\end{array}$ & Grupo & Sexo & Lateralidade & $\begin{array}{l}\text { Tomo } \\
\text { (última) }\end{array}$ & $\begin{array}{l}\text { QT e No } \\
\text { de ciclos }\end{array}$ & Cirurgia & $\begin{array}{l}\text { Mets pós } \\
\text { Tratamento }\end{array}$ & $\begin{array}{c}\text { Seguimento após } \\
\text { Tratamento }\end{array}$ \\
\hline 25 & Va & M & $\begin{array}{l}\text { Unilateral } \\
\text { OE }\end{array}$ & $\begin{array}{l}\text { Restrito ao } \\
\text { Globo }\end{array}$ & $\begin{array}{l}\text { QT 1 }{ }^{\mathrm{a}} \\
2 \text { ciclos }\end{array}$ & Enucl & Não & 18 meses \\
\hline 18 & $\mathrm{Vb}$ & $\mathrm{F}$ & $\begin{array}{l}\text { Unilateral } \\
\text { OD }\end{array}$ & $\begin{array}{l}\text { Metástase } \\
\text { Crânio }\end{array}$ & $\begin{array}{l}\text { QT 1a } \\
5 \text { ciclos }\end{array}$ & Enucl $^{*}$ & Sim & $\begin{array}{l}8 \text { meses } \\
\text { óbito }\end{array}$ \\
\hline 15 & $\begin{array}{l}\text { OD-IVa } \\
\text { OE-Vb }\end{array}$ & M & Bilateral & $\begin{array}{l}\text { Metástase } \\
\text { Crânio }\end{array}$ & $\begin{array}{c}\text { QT 1a } \\
3 \text { ciclos }\end{array}$ & - & Sim & $\begin{array}{l}12 \text { meses } \\
\text { óbito }\end{array}$ \\
\hline 26 & $\begin{array}{l}\text { OD-Va } \\
\text { OE-Vb }\end{array}$ & $\mathrm{F}$ & Bilateral & $\begin{array}{l}\text { Restrito ao } \\
\text { globo c/ } \\
\text { invasão vítrea }\end{array}$ & $\begin{array}{l}\text { QT 1a } \\
3 \text { ciclos }\end{array}$ & $\begin{array}{c}\text { Enucl } \\
\text { OD-Dez/98 } \\
\text { OE-Jan/00 }\end{array}$ & Não & 18 meses@ \\
\hline 30 & $\begin{array}{l}\text { OD-IIIa } \\
\text { OE-Va }\end{array}$ & M & Bilateral & $\begin{array}{l}\text { Restrito ao } \\
\text { globo cl } \\
\text { invasão vítrea }\end{array}$ & $\begin{array}{l}\text { QT 1a } \\
2 \text { ciclos }\end{array}$ & $\begin{array}{l}\text { Enucl } \\
\text { OD }\end{array}$ & Não & $\begin{array}{l}6 \text { meses @ } \\
\text { TTT-3 sessões }\end{array}$ \\
\hline 28 & Va & M & Unilateral OD & $\begin{array}{l}\text { Metástase } \\
\text { Crânio }\end{array}$ & $\begin{array}{l}\text { QT Adj } \\
2 \text { ciclos }\end{array}$ & Enucl $^{*}$ & Não & $\begin{array}{l}2 \text { meses } \\
\text { óbito }\end{array}$ \\
\hline 38 & $\begin{array}{l}\mathrm{OD}-\mathrm{Vb} \\
\mathrm{OE}-\mathrm{Vb}\end{array}$ & $\mathrm{F}$ & Trilateral & $\begin{array}{l}\text { Metástase } \\
\text { Crânio }\end{array}$ & $\begin{array}{l}\text { QT Adj } \\
5 \text { ciclos }\end{array}$ & $\begin{array}{c}\text { Enucl }^{* *} \\
\text { AO }\end{array}$ & Não & $\begin{array}{l}7 \text { meses } \\
\text { óbito }\end{array}$ \\
\hline \multicolumn{9}{|c|}{$\begin{array}{l}\text { Grupo=estadiamento de Reese-Ellsworth para retinoblastoma, M=masculino, F=feminino, QT=quimioterapia, №=número, Enucl=enucleação, Mets=metástases, } \\
\text { TTT=termoterapia trans-pupilar } \\
\text { *Pacientes enucleados com limites comprometidos no exame anatomopatológico. @ Pacientes fizeram radioterapia (EBRT) no olho menos comprometido. } \\
\text { Grupo I- pacientes } 1 \text { a 5. Figuras:1, 2, 3. Grupo II- pacientes } 6 \text { e } 7 \text {. }\end{array}$} \\
\hline
\end{tabular}



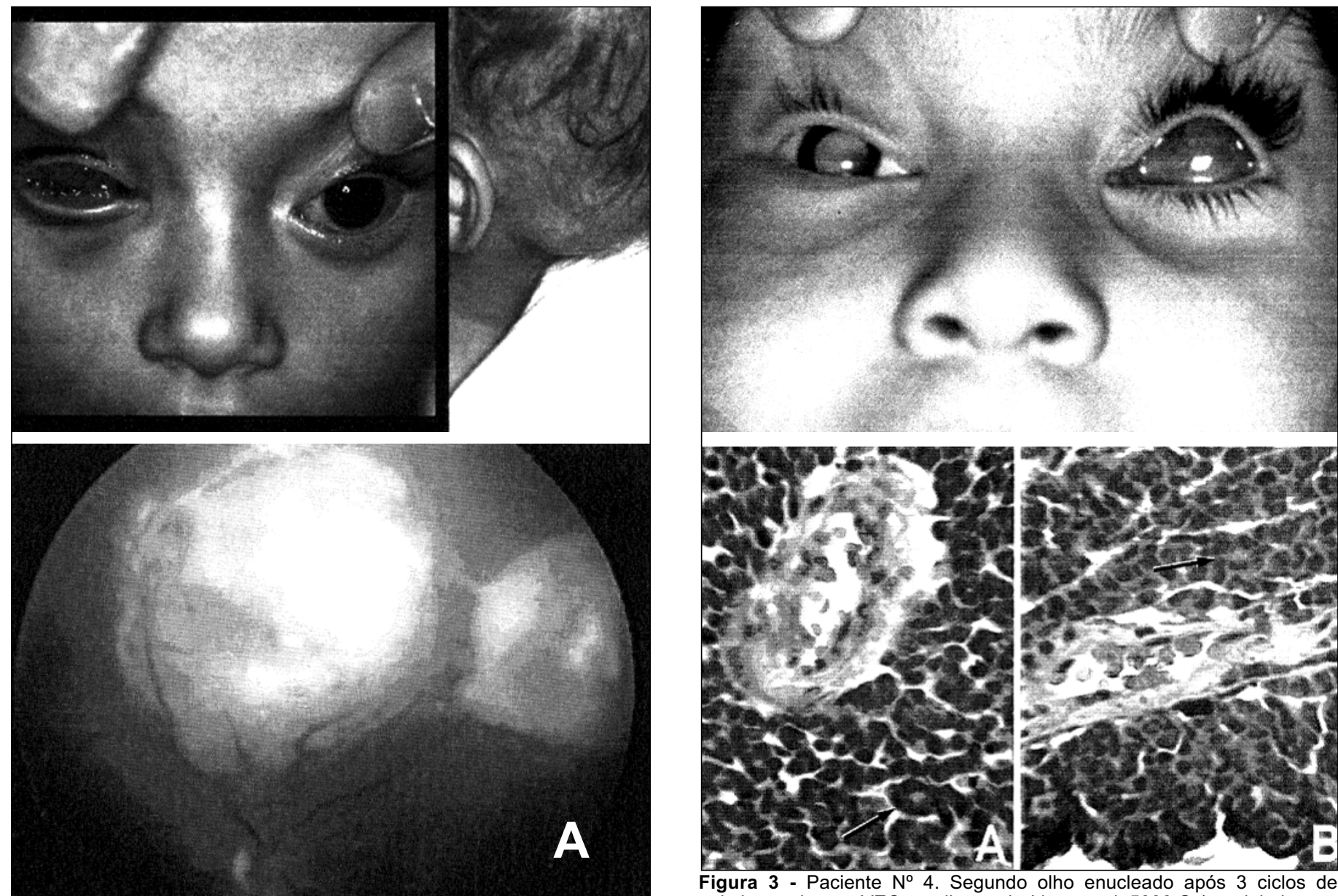

Figura 3 - Paciente $\mathrm{N}^{\circ} 4$. Segundo olho enucleado após 3 ciclos de quimioterapia com VEC e radioterapia (dose total $=5000 \mathrm{~Gy}$ ) evoluindo com descolamento de retina em funil, neovasos de íris e olho cego doloroso. A. No anátomo-patológico apresentou células tumorais viáveis em rosetas (flechas), invasão peri-vascular. B. Corte anatomo-patológico do mesmo olho, na altura do corpo ciliar releva invasão desta estrutura por células viáveis. O nervo óptico apresentou margens cirúrgicas livres.

retina em funil com fibrose e calcificação após a radioterapia. $\mathrm{O}$ anatomo-patológico revelou que mesmo o segundo olho enucleado continha células tumorais viáveis (Fig. 3.A) e invasão do corpo ciliar (Fig. 3.B). Grupo II- Dois pacientes submetidos a enucleação primária com quimioterapia adjuvante, média de 3 ciclos (variando de 2 a 5 ciclos). Estes já apresentavam metástase cerebral e foram a óbito em média aos 4,5 meses de seguimento. Esse grupo foi submetido a enucleação imediatamente após diagnóstico por serem casos avançados com comprometimento extra-ocular e grande volume tumoral. A intervenção cirúrgica teve como objetivo diminuir a massa tumoral a ser tratada.

6 - Dos 8 olhos enucleados 4 tinham invasão do nervo óptico pelo tumor no exame anatomopatológico $(50 \%)$ e margens cirúrgicas comprometidas apesar do menor coto (de nervo óptico) medido ter sido $10 \mathrm{~mm}$.

7 - Dos 7 pacientes estudados, 4 evoluíram para o óbito (2 casos unilaterais, 1 bilateral e 1 trilateral), todos devido a metástases cranianas. A sobrevida média dos pacientes que

evoluíram para óbito foi de 7,2 meses após início do tratamento com quimioterapia. Todos os pacientes estudados apresentavam pelo menos um olho com classificação de ReeseEllsworth grupo V portando acreditamos que o dado determinante para a sobrevida tenha sido a presença de doença extraocular.

\section{DISCUSSÃO}

Semelhante a publicações científicas anteriores, o retinoblastoma não apresenta predileção por sexo. A história familiar obtida em todos os casos estudados foi negativa $(100 \%)$. Apesar de ser uma amostra pequena, segue padrões de incidência de casos esporádicos $(94 \%)$ descritos ${ }^{5}$.

Quanto à lateralidade, a freqüência encontrada não foi compatível com o descrito para a doença (30\% bilaterais) $)^{6,17}$ uma vez que foram eliminados os casos não tratados com quimioterapia. Observamos um elevado número de pacientes em estágio avançado da doença, demostrando proptose em 
$25 \%$ dos casos. Tal fato está diretamente relacionado à elevada média de idade de chegada ao nosso hospital $(25,7$ meses), enquanto nos EUA a média é de 18 meses de idade ${ }^{17}$. Todos os sinais e sintomas da doença foram notados pelos familiares em contraste com da literatura americana onde 50\% dos casos são observados pelos pediatras ${ }^{4}$.

Neste estudo destaca-se a alta incidência de metástase detectada pela tomografia e margens cirúrgicas comprometidas do N.O na enucleação apesar de serem retiradas margens amplas, todas maiores ou iguais a $10 \mathrm{~mm}$. Todos estes casos evoluíram para óbito.

Relatos de grandes centros dos E.U.A indicam que pacientes com extensão extra-ocular têm recorrência em 10 a $12 \%$ dos casos ${ }^{10}$. Merriam ${ }^{11}$ relatou sobrevida de 3,5 meses para pacientes com doença extra-ocular e Reese ${ }^{12}$ decreveu 9 casos de sobrevida (de grupo de 25 pacientes) tratados com exenteração e radioterapia. Observamos certa limitação no tratamento dos pacientes com extensão extra-escleral, todas diagnosticadas com mais de 15 meses de idade. O plano terapêutico inicial envolvia associação com radioterapia (EBRT) mas devido às condições nutricionais deficitárias destas crianças, o tratamento com quimioterapia foi complicado e arrastado com desfecho letal.

No passado, a quimioterapia era reservada para tumores invadindo nervo óptico e órbita e com metástases cerebrais. Recentemente, a quimioterapia tem surgido como a grande arma no tratamento do retinoblastoma, particularmente no manejo de doença intra-ocular. Protocolo de quimioterapia com VEC tem surtido efeito terapêutico significativo para redução tumoral, possibilitando tratamento adjuvante mais conservador ${ }^{9}$. A penetração dessa QT intravenosa no vítreo e no espaço subretineano são evidentes e foi relatada em estudo experimental, em olhos normais de primatas, comparando níveis intra-oculares de carboplatina e etoposide demostrou níveis mensuráveis apenas de carboplatina quando usada intravenosa e principalmente em uso peribulbar e episcleral ${ }^{13}$.

No presente estudo, observou-se que o protocolo com VEC, com uma média de 3 ciclos, obteve regressão do volume tumoral no grupo I, num seguimento médio de 12,4 meses ( 5 pacientes tratados com quimioterapia primária); dois responderam apenas inicialmente ao tratamento, e apresentaram metástase cerebral após a quimioterapia. Os pacientes foram enucleados quando pelo menos metade do olho estava preenchido por tumor. Observou-se em série de casos que metástases que surgem após tratamento inicial com quimioterapia tem menor resposta ao tratamento ${ }^{14,15}$. Os pacientes submetidos à radioterapia (EBTR) obtiveram um melhor controle local da doença apesar de um deles ter evoluído para enucleação devido a descolamento de retina em funil e olho cego/doloroso.

No grupo II os 2 pacientes enucleados que já apresentavam metástases cerebrais inicialmente foram submetidos a quimioterapia adjuvante com VEC (média de 3 ciclos) para tratamento das metástases. A resposta a quimioterapia foi pequena e a sobrevida média de 4,5 meses; ambos foram a óbito. Namouni ${ }^{16}$, também observou em seu trabalho que pacientes com comprometimento do SNC não obtiveram alteração da evolução da doença através do uso de altas doses de QT. Dunkel e colaboradores desenvolveram um protocolo com altas doses de quimioterapia (carboplatina, tiotepa com ou sem etoposide), transplante autólogo de medula e radioterapia e obtiveram sobrevida em seus 4 casos tratados durante mais de 46 meses $^{19}$.

A sobrevida e o padrão metastático de pacientes com doença metastática foi relatada e a sobrevida média observada foi 5,8 meses $^{20}$. Em nossa série, aqueles inicialmente diagnosticados com metástases apresentaram sobrevida média de 4,5 meses ( $\operatorname{casos} 6$ e 7).

A complicação mais temida da quimioterapia com VEC é o risco de desenvolver outros tumores a médio e longo prazo, em especial a leucemia pelo uso do Etoposide ${ }^{9}$. A radioterapia parece apresentar maior incidência de tumores não-oculares ${ }^{21}$. $\mathrm{O}$ efeito e as complicações tardias não foram observados em nossos pacientes devido ao curto período de seguimento.

\section{CONCLUSÕES}

Este estudo tem limitações por não ser controlado, pela pequena amostra e curto período de seguimento considerando-se uso de protocolo de quimioterápico. Por esta razão, optou-se por não fazer comparações estatísticas em termos de sobrevida e complicações do tratamento.

Entretanto pode-se concluir que: 1 -os casos de retinoblastoma são diagnosticados tardiamente em nosso meio e suas apresentações clínicas avançadas pioram o prognóstico dos pacientes. 2- A porcentagem de enucleações com margens livres neste grupo foi $50 \%$, metade dos casos. 3- A quimioterapia com protocolo utilizando $\mathrm{VEC}$ associado ou não a radioterapia ou enucleação demostrou redução tumoral satisfatória e melhora da sobrevida dos pacientes com doença avançada, embora não houve tratamento eficaz que alterasse o desfecho letal da doença quando existe metástase craniana e/ou invasão de nervo óptico. Estudos randomizados serão necessários para determinar a eficácia e complicações dos protocolos quimioterápicos usados no Brasil.

\section{SUMMARY}

Purpose: Retrospective uncontrolled series of consecutive patients with retinoblastoma considering: 1-tumor features on computed tomography, 2-proportion of patients with optic nerve invasion in pathology studies of enucleated eyes and 3-results after chemotherapy.

Methods: The authors revised the charts of 11 consecutive patients diagnosed with retinoblastoma between Febuary/ 98 and September/99, treated at the Departments of Pediatric Oncology and Ophthalmology. Seven cases who 
received chemotherapy with vincristine sulfate, etoposide and carboplatin (VEC) were selected for this study to evaluate tumor presentation (response to chemotherapy and patient survival).

Results: The seven studied patients were diagnosed between the ages of 15 and 38 months (mean =25.7 months), 3 being unilateral, 3 bilateral and 1 trilateral (with pinealoblastoma). All patients were treated with VEC protocol administered in 2 to 5 cycles, and divided in to 2 groups: Group1- Patients treated with primary chemotherapy to obtain tumor reduction and preservation of one of the eyes (5 patients); Group2- Patients treated with aggressive chemotherapy for extraocular disease (2 patients). Of the 5 patients treated with primary chemotherapy (4 subsequently enucleated due to eye filling tumor), 4 achieved some tumor reduction, however 2 of them had only an initial tumor response and died on average 10 months later, one was chemoresistant. Patients with bilateral tumor were also treated with external beam radiation treatment (EBRT) of the eye containing the smaller tumor. The average follow-up period after primary chemotherapy was 12.4 months. The two patients submitted to primary enucleation and adjuvant chemotherapy (Group 2) had brain metastasis and died on average 4.5 months later regardless of treatment.

Conclusion: This study revealed that: 1-retinoblastoma is a disease of late diagnosis and advanced clinical presentation in our area. 2-The percentage of enucleations with free optic nerve margins was only $50 \%$ of the cases. 3-The chemotherapy protocol using VEC associated either with radiotherapy or enucleation provided satisfactory tumor reduction and increased survival in patients with advanced disease but did not change the lethal outcome when brain metastasis and optic nerve invasion were present. Randomized studies will be necessary to determine efficacy and complications of these new chemotherapy protocols.

Abbreviations: $V E C$ - vincristine sulfate $1.5 \mathrm{mg} / \mathrm{m}^{2}$, etoposide $100 \mathrm{mg} / \mathrm{m}^{2}$, carboplatin $360 \mathrm{mg} / \mathrm{m}^{2}$.

Keywords: Retinoblastoma: chemotherapy; Therapy.

\section{REFERÊNCIAS BIBLIOGRÁFICAS}

1. Murphree Al, Munier FL. Retinoblastoma. Ryan SJ, ed. Retina. 2 $2^{\text {ed }}$ St Louis: Mosby-Year, 1994;.605-6,v.1, capítulo 27.

2. Abramsom DH, Niksarli, Ellsworth RM. Changing trends in the management of retinoblastoma: 1951-1965 vs. 1966-1980. J Pediatr Ophthalmol Strabismus 1994;31:32-7.

3. Shields JA \& Augsburger JJ: Current approaches to the diagnosis and management of retinoblastoma. Surv Ophthalmol 1981;25:347-71.

4. Amoaku WMK, Willshaw HE, Parkes SE, Shah KJ, Mann JR. Trilateral retinoblastoma. A report of five patients. Cancer 1996;78:858-63.

5. Jansen RD, Miller RW. Retinoblastoma. Epidemiologic characteristics. N Engl J Med 1971;285:307-11.

6. Shields JA, Shields CL, Sivalingan V. Decrecing frequency of enucleation in patients with retinoblastoma. Am J Ophthalmol 1989;108:185-8.

7. The Commitee for National Registry of Retinoblastoma. Ophthalmic Surg 1980;11:596-8.

8. Perez, Carlos A.Principles and Practice of Radiation Oncology $3^{\text {a }}$ ed. - Philadelphia / New York Lippincott - Raven Publisher, 1995.

9. Shileds CL, Shields JA, Needle M et al. Combined chemoreduction and adjuvant treatment for intraocular retinoblastoma. Ophthalmology 1997;104 (12):2101-11.

10. Doz F, Khelfaoui, Mosseri V, et al. The role of chemotherapy in orbital involvement of retinoblastoma. The experience at a single institution with 33 patients. Cancer 1994;74:722-32.

11. Merriam GR. Retinoblastoma: analysis of seventeen autopsies. Arch Ophthalmol 1950;44:71-108.

12. Reese AB. Tumor of the eye. $2^{\text {nd }}$ ed. New York: Hoeber Medical Division, Harper \& Row 1963;155-6.

13. Mendelsohn ME, Abramsom DH, Madden T et al. Intraocular concentrations of chemotherapeutic agents after systemic or local administration. Arch Ophthalmol 1998;116:1209-12.

14. Kiratli H, Bilgiç S at al. Management of massive orbital involvement of intraocular retinoblastoma. Ophthalmology 1998;105:322-6.

15. With L. Chemoterapy for retinoblastoma: where do we go from here? Symposium on Retinoblastoma, October 1990. Ophathalmic Paediatr Genet 1991;12:115-30.

16. Namouni F, Doz F, Tanguy ML. et al. High-dose chemotherapy with carboplatin, etoposide and cyclophosphamide followed by a haematopoietic stem cell rescue in patients with high-risk retinoblastoma: a SFOP and SFGM study. Europ Jour Cancer 1997 33(14):2368-75.

17. Rubenfeld M, Abramson DH, Ellsworth RM, Kitchin D. Unilateral vs. bilateral retinoblastoma- Correlations between age at diagnosis and stage of ocular disease. Ophthalmology 1986, 93(8):1016-9.

18. Abramson DH, Gombos DS. The topography of bilateral retinoblastoma lesions. Retina 1996,16(3):232-9.

19. Dunkel IJ et al. Treatment of metastatic retinoblastoma. Ophthalmology (no prelo).

20. MacKay CJ, Abramson DH, Ellsworth RM. Metastatic patterns of retinoblastoma. Arch Ophthalmol 1984,102(4):391-6.

21. Abramson DH, Frank CM. Second nonocular tumors in survivors of bilateral retinoblastoma. A possible age effect on radiation-related risk. Ophthalmology 1998;105(4):573-80.

\title{
VII Congresso Sul Brasileiro de Oftalmologia XI Simpósio da Sociedade Catarinense de Oftalmologia I Simpósio Regional Sul da Sociedade Brasileira de Plástica Ocular
}

\author{
30 e 31 de Março de 2.001
}

COSTÃO DO SANTINHO RESORT - FLORIANÓPOLIS - SC

INFORMAC̣ÕES: Fax : (48) 239-4130

e-mail: ivan.coelho@fsb.zzn.com / icoj@ @usa.net 